\title{
Influência do grupo genético, condição sexual e tratamento antiparasitário nas medidas de área de olho do lombo e espessura de gordura in vivo e na carcaça de bovinos de corte
}

[Influence of breed, gender condition, and anti-parasitic treatment on the measurements of in vivo rib eye area and fat thickness and on the carcass of beef cattle]

\author{
R.M.K. Pinheiro ${ }^{1}$, T.J.P. Silva ${ }^{2 *}$, J.H.M. Viana $^{3}$ \\ ${ }^{1}$ Aluna de pós-graduação - UFF - Niterói, RJ \\ ${ }^{2}$ Universidade Federal Fluminense \\ Rua Vital Brasil Filho, 64 \\ 24230-340 - Niterói, RJ \\ ${ }^{3}$ Embrapa Gado de Leite - Juiz de Fora, MG
}

\begin{abstract}
RESUMO
Foram estudados 48 bovinos machos oriundos de inseminação artificial, criados em pasto, sendo 24 (12 Nelore e 12 F1 1/2 Red Angus-Nelore ) tratados com antiparasitários alopáticos e 24 (mesmo número de puros e cruzados) tratados com o antiparasitário bioterápico Fator C\&MC. Os animais foram desmamados aos oito meses, metade de cada subgrupo genético (6) foi castrado aos 13 meses e todos abatidos aos 32 meses, com o objetivo de verificar a influência do tratamento antiparasitário, do grupo genético e da condição sexual sobre as medidas de área de olho de lombo (AOL) e espessura de gordura de lombo (EGL). Usaram-se medidas de ultrassonografia no animal vivo (AOLU e EGLU) e na carcaça, plástico quadriculado e paquímetro (AOLC e EGLC). Os animais $\mathrm{F} 1$, os inteiros e os tratados com alopatia apresentaram peso vivo maior quando comparados aos Nelores, castrados e tratados com bioterápicos. Não houve diferença da AOLU e AOLC entre os grupos genéticos. EGLC foi mais alta nos cruzados. Os animais inteiros apresentaram AOLU e AOLC maiores que os castrados, e EGLU e EGLC menores. Foram altamente significativos os coeficientes de correlação entre as medidas por ultrassom e na carcaça para área de olho de lombo $(0,87)$ e espessura de gordura do lombo $(0,95)$.
\end{abstract}

Palavras-chave: bovino, bioterápico, castração, carcaça, ultrassonografia

\begin{abstract}
Forty-eight male bovines, products of artificial insemination and raised on pasture, were studied, being 24 (12 Nelore, $12 \mathrm{~F} 11 / 2$ Nelore $1 / 2$ Red Angus) treated with allopathic antiparasitic drugs and 24 (same number of pure and crossbred) treated with a biotherapic antiparasitic drug Factor C\&MC. Animals were weaned at eight months of age and half of each genetic subgroup (six) was castrated at 13 months of age. All animals were slaughtered at 32 months of age, in an attempt to evaluate the influence of antiparasitic treatment, genetic group, and gender condition in the measurements of rib eye area (AOL) and fat thickness (EGL) of loin. Measurements of ultrasonography were used for live animals (AOLU and EGLU), whereas a direct plastic grid reading of the eye muscle and a pachymeter (AOLC and EGLC) were used for carcasses. F1 animals, noncastrated, and those treated with allopathic drugs showed higher live weight when compared with Nelore, castrated, and biotherapic treated animals. There were no differences between genetic groups of AOLU and AOLC. EGLC was higher in crossbred animals. Non-castrated animals showed higher AOLU and AOLC when compared with castrated animals, and lower EGLU and EGLC. Correlation coefficients for ultrasound and carcass measurements were highly significant for rib eye area (0.87) and fat thickness (0.95).
\end{abstract}

Keywords: bovine, biotherapic, castration, carcass, ultrasound

Recebido em 4 de agosto de 2008

Aceito em 6 de maio de 2009

*Autor para correspondência (corresponding author)

E-mail: romakapin@gmail.com 


\section{INTRODUÇÃO}

Há uma tendência mundial para produção de produtos orgânicos e, no caso da criação de bovinos, uma das condições exigidas é não utilizar no manejo antiparasitário medicamentos convencionais, que são substituídos por tratamentos alternativos, como homeopatia, fitoterapia e acupuntura.

Os bioterápicos são preparações medicamentosas de uso homeopático, obtidos a partir de produtos biológicos, quimicamente indefinidos como secreções, excreções, tecidos e órgãos, produtos de origem microbiana e alérgenos (Farmacopeia..., 1997).

A determinação da composição corporal de bovinos de corte é importante para avaliar o desempenho, quando se tem como objetivo a produção de carcaças com maior proporção de músculos e quantidades adequadas de gordura, visando atender às exigências do mercado consumidor. $\mathrm{O}$ ganho de peso é a medida mais utilizada para avaliar o crescimento animal, embora durante o crescimento possam ocorrer variações no peso e no tamanho dos animais, bem como mudanças nas proporções dos tecidos depositados. Muniz e Queiroz (1999) e Arrigoni et al. (2002) demonstraram as diferenças entre os cruzamentos quanto ao crescimento dos tecidos e precocidade em depositar tecido adiposo em local adequado às características desejáveis da carne bovina. A avaliação da espessura de gordura subcutânea e da área de olho de lombo, cuja medida é feita entre a $12^{\mathrm{a}}$ e a $13^{\mathrm{a}}$ costela, tem sido aceita e utilizada como indicadora da composição da carcaça (Figueiredo et al., 2000).

Porto et al. (2000) concluíram que a castração não prejudica o desempenho de animais cruzados, até os 18 meses de idade, mantidos em pasto, e que a integridade dos testículos em animais cruzados proporciona melhores resultados que os observados em castrados. O mesmo se pode dizer do rendimento e do peso de carcaça quente e da relação carne/gordura.

Têm-se verificado maiores peso de abate, rendimento de carcaça e proporção de músculo na carcaça de animais inteiros e maior proporção de gordura na carcaça e marmoreio nos animais castrados (Restle et al., 1996; Ribeiro et al., 2002; Vittori et al., 2007).
A ultrassonografia tem sido utilizada para avaliar as características de carcaça em bovinos vivos, já que permite uma avaliação rápida, não invasiva ou destrutiva, com boa precisão da composição corporal. Vários trabalhos mostram alta correlação entre as medidas realizadas por ultrassom pré-abate e as respectivas medidas na carcaça (Rouse et al., 1992; Luz e Silva, et al., 2003; Prado et al., 2004; Tarouco et al., 2005). Suguisawa (2002) observou efeito significativo do grupo genético para área de olho de lombo apenas quando mensurado na carcaça. Quando a medição foi feita por ultrassonografia, esse efeito não foi significativo.

Esta pesquisa foi desenvolvida com o objetivo de estudar a utilização da ultrassonografia, comparando a influência de raças, da condição de castrado ou inteiro e do tratamento antiparasitário (TAP) com produtos alopático e bioterápico, na avaliação do peso vivo no abate, da área de olho do lombo (AOLU) e da espessura de gordura do lombo (EGLU) de bovinos vivos, e correlacionar essas medidas com as obtidas na carcaça (AOLC e EGLC).

\section{MATERIAL E MÉTODOS}

Foram utilizados 48 bovinos machos, filhos de vacas Nelores comuns, inseminadas com sêmen de Nelore e de Red Angus, nascidos e recriados na fazenda Paraíso, município de Valença, RJ.

O delineamento experimental foi inteiramente ao acaso, em esquema fatorial $2 \times 2 \times 2$ (dois grupos genéticos: Nelore e 1/2 Red Angus-Nelore; duas condições sexuais: inteiro e castrado; e dois tratamentos antiparasitários: TAP- alopatia e TAP-bioterápico) e seis repetições.

A partir da divisão do início do experimento, o grupo TAP-alopático foi tratado contra ecto e endoparasitos (moscas, bernes, carrapatos e verminoses) durante todo o experimento, com medicamentos convencionais da fazenda. Por sua vez, o grupo TAP-bioterápico recebeu apenas o bioterápico comercial (Fator C\&MC) ${ }^{1}$ (para o controle de vermes, moscas, carrapatos e bernes), misturado no sal mineral de maneira constante, seguindo as indicações do fabricante.

${ }^{1}$ Laboratório Fauna e Flora Arenales Ltda.- Presidente Prudente, Brasil. 
Ambos os grupos receberam as vacinas do manejo convencional da fazenda, nos períodos normais de campanha. Nessas ocasiões, somente o grupo TAP-alopático foi tratado com produtos pour-on e com vermífugos, estes estrategicamente no início da seca (maio-junho) e na entrada das águas (setembro-outubro), com uma aplicação adicional no meio do período seco ou sempre que necessário, de acordo com os exames de fezes.

Os animais foram castrados aos 13 meses, de acordo com a sua distribuição no experimento. $\mathrm{Na}$ última pesagem na fazenda antes do abate, aos 32 meses, os animais foram avaliados quanto à AOLU e à EGLU por ultrassonografia ${ }^{2}$. Após a imobilização do animal em um tronco de contenção, localizou-se, por palpação, o espaço entre a $12^{\mathrm{a}}$ e a $13^{\mathrm{a}}$ costela. Sobre o local da medida, foi colocado óleo vegetal para permitir uma boa transmissão e recepção das ondas de ultrassom. As imagens obtidas foram gravadas em um microcomputador acoplado ao ultrassom, e os valores das medidas anotados em planilhas específicas (Fig. 1).

Os bovinos ficaram em repouso, jejum e dieta hídrica por 15 horas antes do abate, de acordo com as normas do RIISPOA (Regulamento..., 1997).

Após o abate e de acordo com as normas da Inspeção Estadual, nas meias carcaças, pesadas, resfriadas por 24 horas e novamente pesadas, foram realizados os cortes primários de dianteiro com cinco costelas, ponta de agulha e traseiro especial. Em seguida, foi realizado o corte transversal para expor o músculo longo dorsal (Longissimus dorsi) do traseiro especial na altura da $12^{\mathrm{a}}$ e da $13^{\mathrm{a}}$ costela, para mensuração da AOLC e EGLC.

A medida da AOLC foi realizada utilizando-se um plástico quadriculado ${ }^{3}$. com um ponto no centro de cada quadrado, onde cada quadrado valia $1 / 10$ de polegada quadrada (Fig. 2). Contando-se o número de quadrados incluídos na área do músculo e dividindo-se o total por 10 , obteve-se a área do olho do lombo, em polegadas ao quadrado.

${ }^{2}$ Aloka SSD 500, transdutor linear de 5 MHzAloka Co. Tóquio, Japão.

${ }^{3}$ USDA Preliminary Cutability Grade, June 1, 1965.
As mensurações de EGLC foram realizadas com utilização de um paquímetro, sendo a medida tomada no terço superior proximal da curvatura lombar do músculo $L$. dorsi, na mesma altura da medida da área de olho do lombo.

A análise de variância para três fatores foi realizada para testar os efeitos de grupo genético (Nelore e cruzado), condição sexual (inteiro e castrado) e tratamento antiparasitário (alopatia e bioterápico) e suas interações para AOLU, EGLU, AOLC e EGLC. O coeficiente de correlação de Pearson foi usado para medir o grau de associação entre essas variáveis. O critério de determinação de significância adotado foi de 5\%. A análise foi processada pelos softwares $\mathrm{SAS} \circledast 8.2 / 2001$.

\section{RESULTADOS E DISCUSSÃO}

$\mathrm{Na}$ Tab. 1 são apresentados os valores de peso vivo, AOLU, EGLU, AOLC e EGLC, de acordo com o grupamento genético, condição sexual e tratamento antiparasitário.

Animais inteiros e tratados com alopatia apresentaram maior peso vivo $(\mathrm{P}<0.05)$ quando comparados aos F1, castrados e/ou tratados com bioterápico (Tab. 1). Os bovinos inteiros demonstraram maior velocidade de ganho de peso, sendo mais eficientes na transformação de alimentos em peso vivo final, semelhante aos resultados de Restle et al. (1996), Porto et al. (2000) e Vittori et al. (2007). Os animais cruzados obtiveram peso vivo maior $(\mathrm{P}<0,01)$ do que os da raça Nelore, conforme relataram Muniz e Queiroz (1999), quando animais cruzados apresentaram-se mais pesados que os zebuínos puros para pesos ajustados aos 550 dias. De modo geral, quanto maior o peso vivo na mesma idade de abate, maior será o valor esperado da área de olho do lombo e do rendimento de carne na carcaça (Rouse et al., 1992; Restle et al., 1996; Ribeiro et al., 2002; Vittori et al., 2007).

Muitos trabalhos têm demonstrado alta correlação entre as medidas realizadas por ultrassom pré-abate e as respectivas medidas na carcaça (Rouse et al., 1992; Arrigoni et al., 2002; Luz e Silva et al., 2003; Tarouco et al., 2005). 


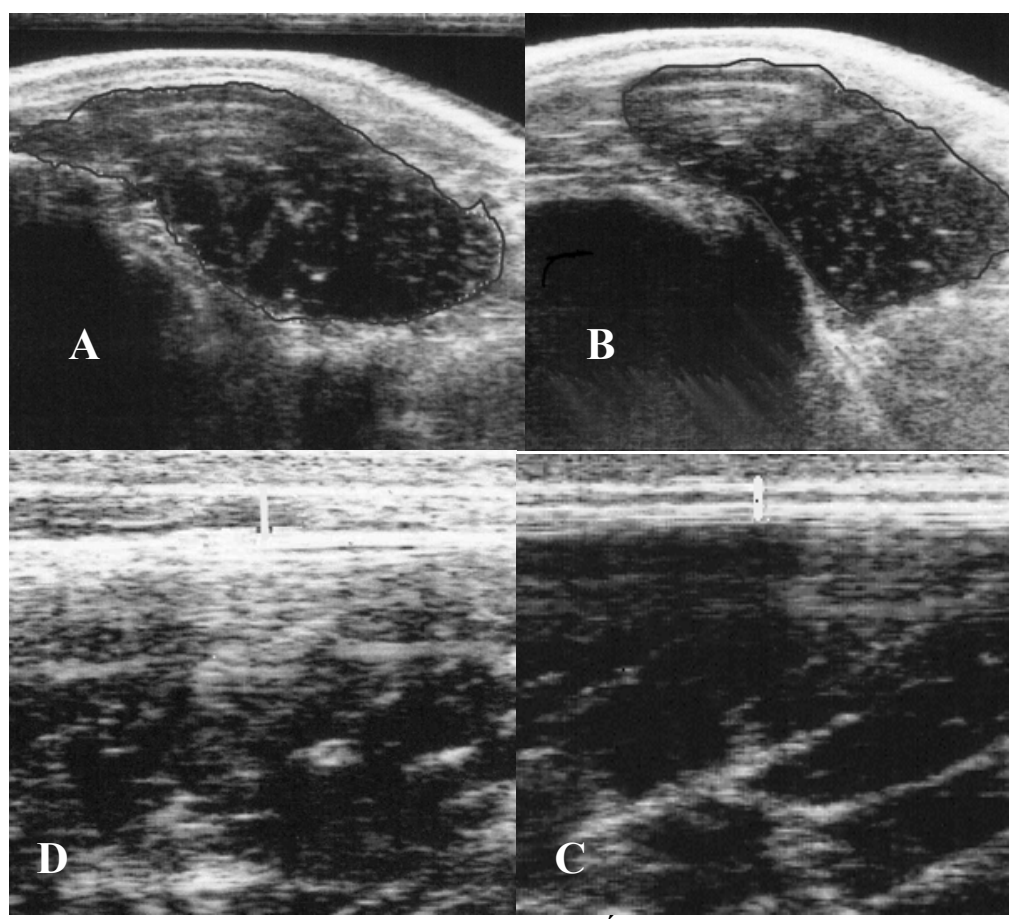

Figura 1. A) Área de olho de lombo de Nelore inteiro; B) Área de olho de lombo de Nelore castrado; C) Espessura de gordura do lombo de Nelore inteiro; e D) Espessura de gordura do lombo de cruzado castrado.

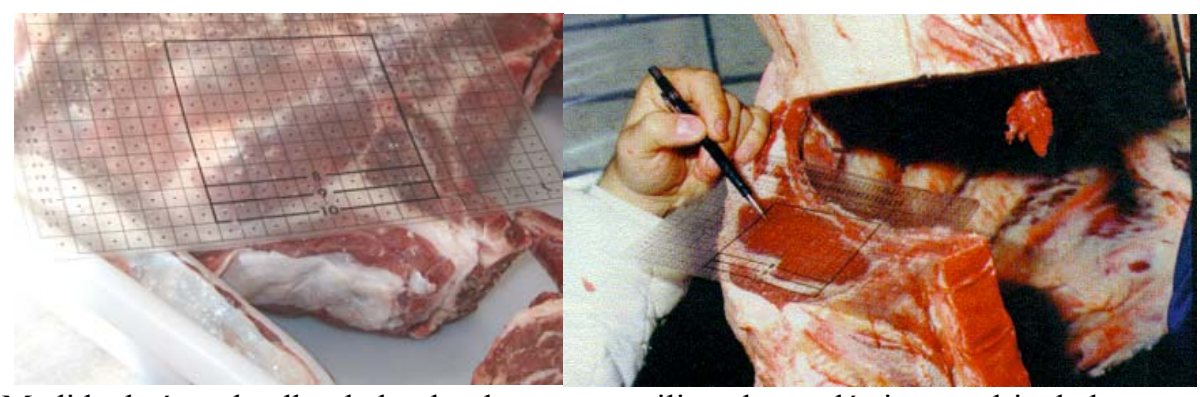

Figura 2. Medida da área de olho de lombo da carcaça utilizando-se plástico quadriculado.

Tabela 1. Valores médios e desvio-padrão do peso vivo, da área do olho de lombo $\left(\mathrm{cm}^{2}\right)$ e espessura de gordura do lombo $(\mathrm{mm})$ medidas por ultrassonografia no animal vivo (AOLU e EGLU) e na carcaça (AOLC e EGLC) de 48 bovinos

\begin{tabular}{|c|c|c|c|c|c|}
\hline \multirow[b]{2}{*}{ Fator } & \multirow{2}{*}{$\begin{array}{c}\text { Peso vivo } \\
(\mathrm{kg})\end{array}$} & \multicolumn{2}{|c|}{ Ultrassom } & \multicolumn{2}{|c|}{ Carcaça } \\
\hline & & $\begin{array}{l}\text { AOLU } \\
\left(\mathrm{cm}^{2}\right)\end{array}$ & $\begin{array}{c}\text { EGLU } \\
(\mathrm{mm})\end{array}$ & $\begin{array}{l}\text { AOLU } \\
\left(\mathrm{cm}^{2}\right)\end{array}$ & $\begin{array}{l}\text { EGLU } \\
(\mathrm{mm})\end{array}$ \\
\hline \multicolumn{6}{|l|}{ Grupamento genético } \\
\hline Nelore & $471,3 \pm 40,9 b$ & $56,0 \pm 4,2 \mathrm{a}$ & $2,20 \pm 1,3 \mathrm{a}$ & $58,5 \pm 4,4 \mathrm{a}$ & $2,40 \pm 1,2 \mathrm{a}$ \\
\hline Cruzado & $490,8 \pm 41,6 \mathrm{a}$ & $55,3 \pm 5,6 a$ & $2,83 \pm 1,7 \mathrm{a}$ & $60,6 \pm 5,5 \mathrm{a}$ & $3,20 \pm 1,7 b$ \\
\hline \multicolumn{6}{|l|}{ Condição sexual } \\
\hline Inteiro & $500,9 \pm 42,3 \mathrm{a}$ & $57,9 \pm 4,2 \mathrm{a}$ & $1,68 \pm 0,8 b$ & $62,2 \pm 4,6 \mathrm{a}$ & $1,92 \pm 0,8 b$ \\
\hline Castrado & $461,2 \pm 31,5 b$ & $53,3 \pm 4,5 b$ & $3,42 \pm 1,59 \mathrm{a}$ & $56,9 \pm 4,0 \mathrm{~b}$ & $3,68 \pm 1,5 a$ \\
\hline \multicolumn{6}{|c|}{ Tratamento antiparasitário } \\
\hline Produto alopático & $504,3 \pm 39,2 \mathrm{a}$ & $56,2 \pm 4,7 \mathrm{a}$ & $2,97 \pm 1,7 \mathrm{a}$ & $60,1 \pm 4,6 \mathrm{a}$ & $3,10 \pm 1,54 \mathrm{a}$ \\
\hline Produto bioterápico & $457,8 \pm 30,7 \mathrm{~b}$ & $55,1 \pm 5,1 \mathrm{a}$ & $2,13 \pm 1,3 b$ & $59,0 \pm 5,5 \mathrm{a}$ & $2,50 \pm 1,4 \mathrm{~b}$ \\
\hline
\end{tabular}

Médias na coluna, seguidas por letras distintas, diferem entre si $(\mathrm{P}<0,05)$. 
Os fatores grupamento genético e tratamento antiparasitário não influenciaram a $\mathrm{AOLU}$ e a AOLC, mas houve diferença significativa devido à condição sexual, pois os animais inteiros apresentaram valores de $\operatorname{AOLU}\left(57,9 \mathrm{~cm}^{2}\right)$ e AOLC $\left(62,2 \mathrm{~cm}^{2}\right)$ mais elevados $(\mathrm{P}<0,001)$ que os castrados (Tab. 1).

Ao compararem animais mestiços Nelore com duas raças paternas (uma continental e outra britânica) submetidas a dois tipos de dieta, Ribeiro et al. (2002) não observaram diferenças entre as respectivas áreas de olho de lombo medidas por ultrassonografia. Suguisawa et al. (2003), ao trabalharem com bovinos jovens $1 / 2$ Angus, 1/2 Nelore; 1/2 Canchim 1/2Nelore; 1/2 Simental $1 / 2$ Nelore e Nelore, observaram efeito significativo do grupo genético para área de olho de lombo apenas quando mensurado na carcaça. Quando a medição foi feita por ultrassonografia, esse efeito não foi significativo.

Neste experimento, houve influência dos fatores condição sexual e tratamento antiparasitário sobre a EGLU e a EGLC. O fator grupamento genético não influenciou essa medida quando feita por ultrassonografia, mas, na avaliação da carcaça, os animais cruzados (mais pesados) apresentaram maior espessura de gordura de lombo. Conforme descreveram Figueiredo et al. (2000), essa diferença pode se devido à menor espessura da gordura de cobertura na raça Nelore e outras raças zebuínas, em que há uma tendência de maiores erros de medição quando feita por ultrassonografia. Embora a técnica de ultrassonografia para mensuração da AOL e EGS esteja praticamente consolidada no meio científico, pesquisas demonstram resultados distintos na sua exatidão (Suguisawa, 2002; Tarouco et al., 2005). Os resultados deste trabalho assemelham-se aos obtidos por Prado et al. (2004), quando concluíram que o processo de castração não afeta as medidas de área de olho de lombo, porém apresenta efeito significativo sobre a espessura de gordura. A raça Nelore diferiu das demais, provavelmente por estar com peso abaixo do ideal para o abate. $\mathrm{O}$ fator peso ao abate mostrou-se significativo nos trabalhos realizados por Restle et al. (1996) e Ribeiro et al. (2002).

Os coeficientes de correlação entre as medidas por ultrassom e na carcaça para a área de olho de lombo $(0,87)$ e espessura de gordura subcutânea
$(0,95)$ foram altamente significativos. Este resultado assemelha-se aos de Arrigoni et al. (2002), que encontraram correlação elevada entre as duas medidas de AOL $(0,80)$, e de Luz e Silva et al. (2003), em que as medidas de carcaça obtidas por ultrassom foram altamente correlacionadas com as medidas na carcaça. A correlação entre AOLU e EGSU foi de 0,83 e entre AOLC e EGSC, de 0,86, indicando que a ultrassonografia pode ser um bom método para estimar as características de área de olho de lombo in vivo. Os coeficientes de correlação simples entre as características medidas por ultrassom e espessura de gordura subcutânea e área do músculo Longissimus de carcaças bovinas foram de 0,95 e 0,97 , respectivamente.

Rouse et al. (1992) observaram correlação de 0,86 entre AOLU e AOLC, e 0,91 entre EGSU e EGSC. Entretanto, Ribeiro et al. (2002) relataram correlação de apenas 0,51 entre as duas medidas.

Valores relativamente baixos de medidas de AOL, tanto por ultrassonografia como na carcaça, podem indicar variações devido ao posicionamento do transdutor, a problemas na limpeza do local de tomada da imagem, à interpretação da imagem obtida pelo técnico e a mudanças na configuração dos diferentes tecidos decorrentes do rigor mortis (Suguisawa, 2002).

\section{CONCLUSÕES}

As medidas de carcaças bovinas obtidas por ultrassonografia foram altamente correlacionadas com as medidas na carcaça para AOL e EGL, confirmando o grau de exatidão na estimativa do mérito individual de carcaça dos animais vivos. Os fatores grupamento genético e tratamento antiparasitário não influenciaram a AOLU e a AOLC, mas houve diferença significativa devido à condição sexual, pois os animais inteiros apresentaram valores mais elevados de AOL que os castrados, e estes maiores valores de EGL, ou seja, carne de melhor qualidade comercial.

\section{REFERÊNCIAS BIBLIOGRÁFICAS}

ARRIGONI, M.B.; GUEDES, S.S.; SILVEIRA, A.C. et al. Desempenho, características de carcaça e qualidade de carne de bovinos de diferentes grupos genéticos no sistema superprecoce. In: REUNIÂO ANUAL DA 
SOCIEDADE BRASILEIRA DE ZOOTECNIA, 39., 2002, Recife. Anais... Recife: SBZ, 2002. CDROM.

FARMACOPEIA homeopática brasileira. 2.ed. São Paulo: Ateneu, 1997.

FIGUEIREDO, L.G.G.; ELER, J.P.; FERRAZ, J.B.S. et al. Componentes de variância para área de olho de lombo espessura de gordura subcutânea. In: SIMPÓSIO NACIONAL DE MELHORAMENTO ANIMAL, 3., 2000. Anais... Belo Horizonte: FEPMVZ, 2000. p.385387.

LUZ E SILVA, S.; LEME, P.R.; PUTRINO, S.N. et al. Estimativa do peso e do rendimento de carcaça de tourinhos Brangus e Nelore, por medidas de ultrassonografia. Rev. Bras. Zootec., v.32, p.1227-1235, 2003.

MUNIZ, C. A. S. D.; QUEIROZ, S.A. Avaliação das características de crescimento de animais Nelore puros e cruzados no Estado do Mato Grosso do Sul. Rev. Bras. Zootec., v.28, p.713$720,1999$.

PORTO, J.C.A.; FEIJÓ, G.L.D.; SILVA, J.M. et al. Desempenho e características de carcaça de bovinos f1 Pardo Suíço corte x Nelore, inteiros ou castrados em diferentes idades. Bol. Pesq. Embrapa Gado de Corte, n.12, 2000.

PRADO, C.S.; PÁDUA, J.T.; CORRÊA, M.P.C. et al. Comparação de diferentes métodos de avaliação da área de olho de lombo e cobertura de gordura em bovinos de corte. Cienc. Anim. Bras., v.5, p.141-149, 2004.
REGULAMENTO da inspeção industrial e sanitária de produtos de origem animal. Brasília: Ministério da Agricultura, 1997. 241p.

RESTLE, J.; GRASSI, C.; FEIJÓ, G.L.D. Características de carcaça e da carne de bovinos inteiros ou submetidos a duas formas de castração em condições de pastagem. Rev. Soc. Bras. Zootec., v.25, p.334, 1996.

RIBEIRO, F.G.; LEME, P.R.; MEDEIROS BULLE, M.L et al. Características da carcaça e qualidade da carne de tourinhos alimentados com dietas de alta energia' Rev. Bras. Zootec., v.31, p.749-756, 2002.

ROUSE, G.H.; WILSON, D.E.; DUELLO D.A. et al. The accuracy of real-time ultrasound scans taken serially on small-, medium-, and largeframe steers and bulls slaughtered at three endpoints. Ames: Iowa State University, 1992. p.14-19.

SUGUISAWA, L. Ultrassonografia para predição das características e composição da carcaça de bovinos. Piracicaba, 2002. 70f. Dissertação (Mestrado) - Escola Superior de Agricultura "Luiz de Queiroz", Universidade de São Paulo, Piracicaba, SP.

VITTORI, A.; GESUALDI JUNIOR, A.; QUEIROZ, A.C. et al. Desempenho produtivo de bovinos de diferentes grupos raciais, castrados e não-castrados, em fase de terminação. Arq. Bras. Med. Vet. Zootec., v.59, p.1263-1269, 2007. 